\title{
Notes on general SIC-POVMs
}

\author{
Alexey E. Rastegin \\ Department of Theoretical Physics, Irkutsk State University, \\ Gagarin Bv. 20, Irkutsk 664003, Russia, e-mail: alexrastegin@mail.ru
}

\begin{abstract}
An unavoidable task in quantum information processing is how to obtain data about the state of an individual system by suitable measurements. Informationally complete measurements are relevant in quantum state tomography, quantum cryptography, quantum cloning, and other questions. Symmetric informationally complete measurements (SIC-POVMs) form an especially important class of such measurements. We formulate some novel properties and relations for general SIC-POVMs in a finite-dimensional Hilbert space. For a given density matrix and any general SIC-POVM, the so-called index of coincidence of generated probability distribution is exactly calculated. Using this result, we obtain state-dependent entropic bounds for a single general SIC-POVM. Lower entropic bounds are derived in terms of the Rényi $\alpha$-entropies for $\alpha \in[2 ; \infty)$ and the Tsallis $\alpha$-entropies for $\alpha \in(0 ; 2]$. A lower bound on the min-entropy of a SIC-POVM is separately examined. For a pair of general SIC-POVMs, entropic uncertainty relations of the Maassen-Uffink type are considered.
\end{abstract}

Keywords: Rényi entropy, Tsallis entropy, general SIC-POVM, index of coincidence

\section{INTRODUCTION}

The quantum information science uses quantum states and effects as tools for information processing [1]. At the final stage of any protocol, some measurements are required. Hence, we ask how to obtain information about the state of a quantum system. Informationally complete measurements have found to be useful in many issues. Among numerous methods for retrieving information on the state, the informationally complete measurements $[2,3]$ seem to be the most versatile. Especially interesting cases are when the measurement is symmetric [4] or covariant with respect to a group of physical transformations $[5,6]$. Symmetric informationally complete (SIC) measurements are the subject of active research. Despite of simple definition, SIC-POVMs are difficult to construct. Studies of symmetric POVMs are connected with many mathematical problems $[7,8]$. They are intimately related to problem of building a complete set of mutually unbiased bases (MUBs) [9]. Weyl-Heisenberg (WH) covariant SIC-sets of states were examined in [10]. Tight informationally complete measurements were introduced in [11].

In their original version, SIC-POVMs are assumed to be constructed of only rank-one elements. Some concrete examples in low dimensions are discussed in [4]. For quantum tomography, rank-one SIC-POVMs are maximally efficient at estimating the quantum state [4]. The seamy side is that such a measurement erases the original state of the system being measured. These reasons pertain to situation, when some unknown state is the subject of tomography and also post-tomography processing. It is typical in such a case that only a part of the total system is measured through the tomography process. As a rule, there is a trade-off between efficiency of used measurement and disturbance, which will influence on further stages. In this regard, other versions of SIC-POVMs are of interest. An approximate version of rank-one SIC-POVMs were examined in [12]. General
SIC-POVMs with elements of any rank are considered in $[13,14]$. It has recently been shown that general SICPOVMs exist in all dimensions [15]. Explicit constructions for such POVMs and their dual bases have been presented in [15].

The aim of the present work is to study some generic properties of general symmetric informationally complete measurements. The preliminary material is reviewed in Section II. In particular, dual bases of informationally complete POVMs are briefly considered. In Section III, we exactly calculate the so-called index of coincidence of probability distribution obtained with a general SICPOVM and arbitrary state. Uncertainty bounds for a single general SIC-POVM are considered in Section IV. For these purposes, we respectively use the Tsallis and Rényi entropies, including the so-called min-entropy. Within the Tsallis formulation, we obtain uncertainty relations in the case of detection inefficiencies. In Section V, uncertainty relations of the Maassen-Uffink type are obtained for a pair of general SIC-POVMs. In Section VI, we conclude the paper with a summary of results.

\section{NOTATION}

Let $\mathcal{L}(\mathcal{H})$ be the space of linear operators on $d$ dimensional Hilbert space $\mathcal{H}$. By $\mathcal{L}_{\text {s.a. }}(\mathcal{H})$ and $\mathcal{L}_{+}(\mathcal{H})$, we respectively mean the space of Hermitian operators on $\mathcal{H}$ and the set of positive ones. For two operators $\mathrm{X}, \mathrm{Y} \in \mathcal{L}(\mathcal{H})$, their Hilbert-Schmidt inner product is defined by $[16]$

$$
\langle\mathrm{X}, \mathrm{Y}\rangle_{\mathrm{hs}}:=\operatorname{tr}\left(\mathrm{X}^{\dagger} \mathrm{Y}\right) .
$$

The inner product (1) induces the Frobenius norm, also called the Hilbert-Schmidt norm:

$$
\|\mathrm{X}\|_{2}:=\langle\mathrm{X}, \mathrm{X}\rangle_{\mathrm{hs}}^{1 / 2}=\operatorname{tr}\left(\mathrm{X}^{\dagger} \mathrm{X}\right)^{1 / 2}
$$

To each $X \in \mathcal{L}(\mathcal{H})$, we assign positive operator $|X|:=$ $\sqrt{\mathrm{X}^{\dagger} \mathrm{X}}$. The singular values $\sigma_{i}(\mathrm{X})$ of arbitrary $\mathrm{X} \in \mathcal{L}(\mathcal{H})$ 
are defined as the eigenvalues of $|X| \in \mathcal{L}_{+}(\mathcal{H})$. Then the Schatten $q$-norm is introduced for all $q \in[1 ; \infty]$ as

$$
\|\mathrm{X}\|_{q}:=\left(\sum_{i=1}^{d} \sigma_{i}(\mathrm{X})^{q}\right)^{1 / q}
$$

The family (3) gives the trace norm $\|X\|_{1}=\operatorname{tr}|X|$ for $q=1$, the Frobenius norm (2) for $q=2$, and the spectral norm $\|\mathrm{X}\|_{\infty}=\max \left\{\sigma_{i}(\mathrm{X}): 1 \leq i \leq d\right\}$ for $q=\infty$. These norms and relations between them have found to be useful in various questions of quantum information [17-19]. For each $q \in[1 ; \infty]$ and $\mathrm{X}, \mathrm{Y}, \in \mathcal{L}(\mathcal{H})$, we have $[16]$

$$
\|\mathrm{XY}\|_{q} \leq\|\mathrm{X}\|_{\infty}\|\mathrm{Y}\|_{q}
$$

We will used (4) in section V. For all $q>p \geq 1$ and arbitrary $\mathrm{X} \in \mathcal{L}(\mathcal{H})$, we also have

$$
\|\mathrm{X}\|_{q} \leq\|\mathrm{X}\|_{p}
$$

This relation is actually a consequence of theorem 19 of the classical book [20].

A density matrix $\rho \in \mathcal{L}_{+}(\mathcal{H})$ has unit trace, i.e. $\operatorname{tr}(\boldsymbol{\rho})=1$. Generalized quantum measurements are commonly described within the POVM formalism [21]. Let $\mathcal{N}=\left\{\mathrm{N}_{j}\right\}$ be a set of elements $\mathrm{N}_{j} \in \mathcal{L}_{+}(\mathcal{H})$, satisfying the completeness relation

$$
\sum_{j} \mathrm{~N}_{j}=\mathbb{1}
$$

Here, the $\mathbb{1}$ denotes the identity operator on $\mathcal{H}$. This set $\mathcal{N}=\left\{\mathrm{N}_{j}\right\}$ is a positive operator-valued measure (POVM). Consider some POVM with $d^{2}$ elements $\mathbf{N}_{j}$, which satisfy the following two conditions. First, for all $j=1, \ldots, d^{2}$ we have

$$
\left\langle\mathrm{N}_{j}, \mathrm{~N}_{j}\right\rangle_{\mathrm{hs}}=a .
$$

This condition can be rewritten as $\left\|\mathrm{N}_{j}\right\|_{2}=\sqrt{a}$. Second, the pairwise inner products are all symmetrical, namely

$$
\left\langle\mathrm{N}_{j}, \mathrm{~N}_{k}\right\rangle_{\mathrm{hs}}=b \quad(j \neq k) .
$$

Then the POVM $\mathcal{N}=\left\{\mathrm{N}_{j}\right\}$ is a general SIC-POVM. Combining $\langle\mathbb{1}, \mathbb{1}\rangle_{\mathrm{hs}}=d$ with (7) and (8) finally gives the relation [15]

$$
b=\frac{1-a d}{d\left(d^{2}-1\right)} .
$$

Further, we obtain $\operatorname{tr}\left(\mathrm{N}_{j}\right)=d^{-1}$ for all $j=1, \ldots, d^{2}$. Therefore, the value $a$ is the only parameter characterizing the type of a general SIC-POVM. This parameter is restricted as [15]

$$
d^{-3}<a \leq d^{-2}
$$

The lower bound $a=d^{-3}$ is reached in the case $\mathbf{N}_{j}=$ $d^{-2} \mathbb{1}$, which does not give an informationally complete
POVM. The upper bound $a=d^{-2}$ is achieved, if and only if the POVM elements are all rank-one. The latter is actually the case of usual SIC-POVMs, when each element is represented in terms of the corresponding unit vector as

$$
\mathrm{N}_{j}=d^{-1}\left|\phi_{j}\right\rangle\left\langle\phi_{j}\right|
$$

The formulas (8) and (9) then result in

$$
\left|\left\langle\phi_{j} \mid \phi_{k}\right\rangle\right|=\frac{1}{\sqrt{d+1}} .
$$

In the further discussion, we will usually avoid both the least values of the relation (10). That is, we exclude the case $\mathrm{N}_{j}=d^{-2} \mathbb{1}$ as well as rank-one SIC-POVMs.

In the next sections, we will use the following properties of general SIC-POVMs. First, the elements $\mathbf{N}_{j}$ of a general SIC-POVM form a basis in the space $\mathcal{L}_{\text {s.a. }}(\mathcal{H})$ [15]. Second, each operator $\mathrm{X} \in \mathcal{L}_{\text {s.a. }}(\mathcal{H})$ can be represented in terms of elements of the dual basis as

$$
\mathrm{X}=\sum_{j=1}^{d^{2}}\left\langle\mathrm{~N}_{j}, \mathrm{X}\right\rangle_{\mathrm{hs}} \widetilde{\mathrm{N}}_{j}
$$

Here, the dual basis $\left\{\widetilde{\mathbf{N}}_{j}\right\}$ is a basis in $\mathcal{L}_{\text {s.a. }}(\mathcal{H})$ such that

$$
\left\langle\mathrm{N}_{j}, \widetilde{\mathrm{N}}_{k}\right\rangle_{\mathrm{hs}}=\delta_{j k} \quad \forall j, k \in\left\{1, \ldots, d^{2}\right\}
$$

When some basis in $\mathcal{L}_{\text {s.a. }}(\mathcal{H})$ is formed by positive operators, its dual basis cannot consist of only positive elements $[22,23]$. For a general SIC-POVM $\left\{\mathrm{N}_{j}\right\}$, the dual basis is comprised by operators [15]

$$
\widetilde{\mathbf{N}}_{j}=\frac{d}{a d^{3}-1}\left(\left(d^{2}-1\right) \mathrm{N}_{j}-(1-a d) \mathbb{1}\right) \text {. }
$$

For a usual SIC-POVM with elements (11), the formula (15) is reduced to $\widetilde{N}_{j}=(d+1)\left|\phi_{j}\right\rangle\left\langle\phi_{j}\right|-\mathbb{1}$. Explicit constructions and related properties of general SIC-POVMs are presented in [15].

\section{INDEX OF COINCIDENCE}

In this section, we calculate the so-called index of coincidence of probability distribution generated by a general SIC-POVM on any mixed state. Using indices of coincidence, the writers of [24] derived entropic bounds for a set of several mutually unbiased bases. For usual SICPOVMs, this issue was considered in [25]. The index of coincidence of probability distribution $\left\{p_{j}\right\}$ is defined as $[26,27]$

$$
C(p):=\sum_{j} p_{j}^{2}
$$

This quantity is often called purity [26], when the probabilities are assumed to be eigenvalues of a density matrix. Inverse of (16) is known as the participation number [26]. 
If the pre-measurement state is described by density matrix $\boldsymbol{\rho}, j$-th outcome occurs with the probability

$$
p_{j}(\mathcal{N} \mid \boldsymbol{\rho})=\operatorname{tr}\left(\mathrm{N}_{j} \boldsymbol{\rho}\right) .
$$

For the given SIC-POVM $\mathcal{N}$ and state $\boldsymbol{\rho}$, the quantity $C(\mathcal{N} \mid \boldsymbol{\rho})$ is obtained by substitution of probabilities (17) into (16). The following statement takes place.

Proposition 1 Let general SIC-POVM $\mathcal{N}$ be characterized by the parameter a in the sense of (7). For arbitrary $\boldsymbol{\rho}$, the index of coincidence of generated probability distribution is equal to

$$
C(\mathcal{N} \mid \boldsymbol{\rho})=\frac{\left(a d^{3}-1\right) \operatorname{tr}\left(\boldsymbol{\rho}^{2}\right)+d(1-a d)}{d\left(d^{2}-1\right)} .
$$

Proof. Using (13) and (17), we represent the density matrix in the dual basis as

$$
\boldsymbol{\rho}=\sum_{j=1}^{d^{2}} p_{j} \widetilde{\mathrm{N}}_{j}
$$

For brevity, we put the quantities $\widetilde{a}=\left\langle\widetilde{\mathrm{N}}_{j}, \widetilde{\mathrm{N}}_{j}\right\rangle_{\mathrm{hs}}$ and $\widetilde{b}=$ $\left\langle\widetilde{\mathrm{N}}_{j}, \widetilde{\mathrm{N}}_{k}\right\rangle_{\mathrm{hs}}$ for $j \neq k$. They are calculated by substitution of $\widetilde{\mathrm{N}}_{j}$ and $\widetilde{\mathrm{N}}_{k}$ according to (15). The resulting expressions are then written as

$$
\begin{aligned}
& \widetilde{a}-\widetilde{b}=\frac{d\left(d^{2}-1\right)}{a d^{3}-1} . \\
& \widetilde{b}=\frac{d(a d-1)}{a d^{3}-1},
\end{aligned}
$$

Here, we used $\operatorname{tr}\left(\mathrm{N}_{j}\right)=d^{-1}$, the definitions (7) and (8), and the condition (9). Substituting the right-hand side of (19) into $\operatorname{tr}\left(\boldsymbol{\rho}^{2}\right)$ leads to the formula

$$
\operatorname{tr}\left(\boldsymbol{\rho}^{2}\right)=\widetilde{a} C(p)+\widetilde{b} \sum_{j \neq k} p_{j} p_{k}=(\widetilde{a}-\widetilde{b}) C(p)+\widetilde{b},
$$

where the normalization condition was used at the last step. Combining (22) with (20) and (21) finally leads to the claim (18).

The statement of Proposition 1 gives the expression for the index of coincidence in terms of the parameter $a$ and dimensionality $d$. For the completely mixed state $\boldsymbol{\rho}_{*}=\mathbb{1} / d$ with $\operatorname{tr}\left(\boldsymbol{\rho}_{*}^{2}\right)=d^{-1}$, the formula (18) gives

$$
C\left(\mathcal{N} \mid \boldsymbol{\rho}_{*}\right)=d^{-2},
$$

irrespectively to $a$. The right-hand side of (23) is valid, since $p_{j}\left(\mathcal{N} \mid \boldsymbol{\rho}_{*}\right)=d^{-2}$ for any general SIC-POVM. For a usual SIC-POVMs with only rank-one elements (11), we substitute $a=d^{-2}$ into (18) and obtain

$$
C(\mathcal{N} \mid \boldsymbol{\rho})=\frac{\operatorname{tr}\left(\boldsymbol{\rho}^{2}\right)+1}{d(d+1)} .
$$

The result (24) has been derived in [25] by other method. For pure states, the numerator in the right-hand side of (24) is equal to 2 . This pure-state case of (24) was previously presented in [28]. The method of the paper [28] is based on the fact that the unit vectors $\left|\phi_{j}\right\rangle$ form a spherical 2-design. As was noted in [25], the result (24) is significant from the viewpoint of applications in the entanglement detection. It would be interesting to examine this question with general SIC-POVMs. Indeed, general SIC-POVMs can be built for arbitrary $d$.

In the case $d=2$, the right-hand side of (18) can be represented with use of the Bloch vector. Here, we denote the identity $2 \times 2$-matrix by $\mathbb{1}$ and the usual Pauli matrices by $\boldsymbol{\sigma}_{x}, \boldsymbol{\sigma}_{y}$, and $\boldsymbol{\sigma}_{z}$. Arbitrary density matrix is written as

$$
\boldsymbol{\rho}=\frac{1}{2}(\mathbb{1}+\vec{r} \cdot \vec{\sigma}) .
$$

where $\vec{r}=\left(r_{x}, r_{y}, r_{z}\right)$ is the Bloch vector. Positivity of this matrix implies $r=|\vec{r}| \leq 1$. Calculating $\operatorname{tr}\left(\boldsymbol{\rho}^{2}\right)=$ $\left(1+r^{2}\right) / 2$, the index of coincidence is equal to

$$
C(\mathcal{N} \mid \boldsymbol{\rho})=\sum_{j=1}^{4} p_{j}(\mathcal{N} \mid \boldsymbol{\rho})^{2}=\frac{3+(8 a-1) r^{2}}{12} .
$$

This result shows a dependence of $C(\mathcal{N} \mid \boldsymbol{\rho})$ on the parameter $a$ and the Bloch vector $\vec{r}$. For $a=1 / 4$, the formula (26) gives the fraction $\left(3+r^{2}\right) / 12$, which was already noted in [25]. The Bloch-vector representation for finitelevel systems is one of important state representations [26]. Similarly to (26), the formula (18) could be rewritten in terms of the generalized Bloch vector of a $d$-level system. By $\boldsymbol{\lambda}_{n} \in \mathcal{L}_{\text {s.a. }}(\mathcal{H})$, with $n=1, \ldots, d^{2}-1$, we denote the generators of $\mathrm{SU}(d)$ which satisfy $\operatorname{tr}\left(\boldsymbol{\lambda}_{n}\right)=0$ and

$$
\operatorname{tr}\left(\boldsymbol{\lambda}_{m} \boldsymbol{\lambda}_{n}\right)=2 \delta_{m n} .
$$

The factor 2 in (27) is rather traditional. Arbitrary density operator can be represented in the form [26, 29]

$$
\boldsymbol{\rho}=\frac{1}{d}\left(\mathbb{1}+\sum_{n=1}^{d^{2}-1} r_{n} \boldsymbol{\lambda}_{n}\right)
$$

where $r_{n}=(d / 2) \operatorname{tr}\left(\boldsymbol{\rho} \boldsymbol{\lambda}_{n}\right)$. These components form a $\left(d^{2}-1\right)$-dimensional real vector, which represents the density matrix $\boldsymbol{\rho}$. Although the definition of the Bloch vector is simple, the space of the Bloch vectors for $d$-level system is difficult to determine. Some general properties of the Bloch-vector space are studied in [30-32]. By calculations, we obtain

$$
\operatorname{tr}\left(\rho^{2}\right)=\frac{1}{d}+\frac{2}{d^{2}}\|r\|_{2}^{2},
$$

where $\|r\|_{2}$ denotes the vector 2-norm. The formula (18) is then represented as

$$
C(\mathcal{N} \mid \boldsymbol{\rho})=\frac{1}{d^{2}}+\frac{2\left(a d^{3}-1\right)}{d^{3}\left(d^{2}-1\right)}\|r\|_{2}^{2} .
$$

For $d=2$, this result is reduced to (26). For the completely mixed state $\boldsymbol{\rho}_{*}=\mathbb{1} / d$, components of the generalized Bloch vectors are all zero. Hence, the formula (30) directly leads to (23). Thus, we have useful expressions in terms of the generalized Bloch vector. 


\section{TSALLIS' AND RÉNYI'S FORMULATIONS FOR A SINGLE SIC-POVM}

In this section, we obtain uncertainty relations for a single general SIC-POVM in terms of its Tsallis and Rényi entropies. Entropic functions are convenient tools to measure an uncertainty in quantum measurements $[33,34]$. The Rényi and Tsallis entropies are especially important generalizations of the Shannon entropy. For $\alpha>0 \neq 1$, the Tsallis $\alpha$-entropy of probability distribution $\left\{p_{j}\right\}$ is defined by [35]

$$
H_{\alpha}(p):=\frac{1}{1-\alpha}\left(\sum_{j} p_{j}^{\alpha}-1\right) .
$$

The special case $\alpha=2$ gives the so-called linear entropy $H_{2}(p)=1-C(p)$. The right-hand side of (31) is usually rewritten in terms of the $\alpha$-logarithm

$$
\ln _{\alpha}(x):=\frac{x^{1-\alpha}-1}{1-\alpha}
$$

where $\alpha>0 \neq 1$ and $x>0$. The Tsallis $\alpha$-entropy reads

$$
H_{\alpha}(p)=-\sum_{j} p_{j}^{\alpha} \ln _{\alpha}\left(p_{j}\right)=\sum_{j} p_{j} \ln _{\alpha}\left(\frac{1}{p_{j}}\right) .
$$

In statistical physics, the entropy (31) was originally introduced in [35]. Taking $\alpha \rightarrow 1$, the $\alpha$-logarithm is reduced to the standard logarithm. Then the formula (31) gives the Shannon entropy $H_{1}(p)=-\sum_{j} p_{j} \ln p_{j}$. Functional properties of the entropy (31) and its conditional versions are considered in [36, 37].

For the given SIC-POVM $\mathcal{N}=\left\{\mathrm{N}_{j}\right\}$, the entropy $H_{\alpha}(\mathcal{N} \mid \boldsymbol{\rho})$ is obtained by substituting the probabilities (17) into the formula (31). It turns out that these entropies are bounded from below. We will derive lower bounds on the Tsallis $\alpha$-entropy for $\alpha \in(0 ; 2]$.

Proposition 2 Let general SIC-POVM $\mathcal{N}$ be characterized by the parameter $a$ in the sense of ( 7$)$. For $\alpha \in(0 ; 2]$ and arbitrary density matrix $\boldsymbol{\rho}$, the Tsallis $\alpha$-entropy satisfies the state-dependent bound

$$
H_{\alpha}(\mathcal{N} \mid \boldsymbol{\rho}) \geq \ln _{\alpha}\left(\frac{d\left(d^{2}-1\right)}{\left(a d^{3}-1\right) \operatorname{tr}\left(\boldsymbol{\rho}^{2}\right)+d(1-a d)}\right) .
$$

Proof. The following point was noticed in [25]. For $\alpha \in(0 ; 2]$ and arbitrary probability distribution, the Tsallis $\alpha$-entropy obeys

$$
H_{\alpha}(p) \geq \ln _{\alpha}\left(\frac{1}{C(p)}\right) .
$$

This formula is a direct consequence of Jensen's inequality for the function $x \mapsto \ln _{\alpha}(1 / x)$. Indeed, this function is convex for $\alpha \in(0 ; 2]$. Combining (18) with (35) immediately gives the claim (34).

For all $\alpha \in(0 ; 2]$, the result (34) provides a statedependent lower bound on the Tsallis $\alpha$-entropy of probability distribution generated by a general SIC-POVM.
For $\alpha=2$, the inequality (34) is always saturated. Using (30), we rewrite the bound (34) in terms of the generalized Bloch vector, namely

$$
H_{\alpha}(\mathcal{N} \mid \boldsymbol{\rho}) \geq \ln _{\alpha}\left(\frac{d^{3}\left(d^{2}-1\right)}{2\left(a d^{3}-1\right)\|r\|_{2}^{2}+d\left(d^{2}-1\right)}\right),
$$

where $\alpha \in(0 ; 2]$. For $\alpha=1$, we obtain the lower bound on the Shannon entropy, namely

$$
H_{1}(\mathcal{N} \mid \boldsymbol{\rho}) \geq \ln \left(\frac{d\left(d^{2}-1\right)}{\left(a d^{3}-1\right) \operatorname{tr}\left(\boldsymbol{\rho}^{2}\right)+d(1-a d)}\right) .
$$

With a pure state $\boldsymbol{\rho}=|\psi\rangle\langle\psi|$, the entropic bound (34) is reduced to the inequality

$$
H_{\alpha}(\mathcal{N} \mid \psi) \geq \ln _{\alpha}\left(\frac{d(d+1)}{a d^{2}+1}\right) .
$$

For impure states, we have a stronger lower bound (34). The latter follows from increasing of the $\alpha$-logarithm and and the fact that $\operatorname{tr}\left(\boldsymbol{\rho}^{2}\right)<1$ for an impure state. Here, we see a natural dependence on the measured state. The right-hand side of (34) reaches its maximum with the completely mixed state $\boldsymbol{\rho}_{*}=\mathbb{1} / d$. Using $\operatorname{tr}\left(\boldsymbol{\rho}_{*}^{2}\right)=d^{-1}$, the formula (34) becomes

$$
H_{\alpha}\left(\mathcal{N} \mid \rho_{*}\right) \geq \ln _{\alpha}\left(d^{2}\right) .
$$

The bound (39) is just saturated. Indeed, for all $j=1, \ldots, d^{2}$ we have $\operatorname{tr}\left(\mathrm{N}_{j}\right)=d^{-1}$ and, herewith, $p_{j}\left(\mathcal{N} \mid \boldsymbol{\rho}_{*}\right)=d^{-2}$. Substituting this probability into the right-hand side of (33), we actually obtain (39) with the sign of equality. With the completely mixed state, the equality takes place for all $\alpha>0$ irrespectively to the parameter $a$. In the mentioned sense, the state-dependent bound (34) is tight. At the same time, we proved (34) only for $\alpha \in(0 ; 2]$. Formulating lower bounds on the entropy $H_{\alpha}(\mathcal{N} \mid \boldsymbol{\rho})$ for $\alpha>2$ is an open question.

To consider detection inefficiencies, we will use the following approach [25]. To the given value $\eta \in[0 ; 1]$ and probability distribution $\left\{p_{j}\right\}$, one assigns a "distorted" distribution:

$$
p_{j}^{(\eta)}=\eta p_{j}, \quad p_{\varnothing}^{(\eta)}=1-\eta .
$$

The probability $p_{\varnothing}^{(\eta)}$ corresponds to the no-click event. The parameter $\eta \in[0 ; 1]$ characterizes a detector efficiency. As was shown in the paper [38], for all $\alpha>0$ we have

$$
H_{\alpha}\left(p^{(\eta)}\right)=\eta^{\alpha} H_{\alpha}(p)+h_{\alpha}(\eta) .
$$

Here, the binary Tsallis entropy $h_{\alpha}(\eta)$ is expressed by

$$
h_{\alpha}(\eta):=-\eta^{\alpha} \ln _{\alpha}(\eta)-(1-\eta)^{\alpha} \ln _{\alpha}(1-\eta) .
$$

Such results have been used in studying entropic Bell inequalities with detector inefficiencies [39]. Entropic uncertainty relations with detection inefficiencies for mutually unbiased bases were derived in [25]. Combining (34) 
with (41), for $\alpha \in(0 ; 2]$ we have

$$
\begin{aligned}
H_{\alpha}^{(\eta)}(\mathcal{N} \mid \boldsymbol{\rho}) & \geq \eta^{\alpha} \ln _{\alpha}\left(\frac{d\left(d^{2}-1\right)}{\left(a d^{3}-1\right) \operatorname{tr}\left(\boldsymbol{\rho}^{2}\right)+d(1-a d)}\right) \\
& +h_{\alpha}(\eta)
\end{aligned}
$$

The entropy $H_{\alpha}^{(\eta)}(\mathcal{N} \mid \boldsymbol{\rho})$ is calculated for the distribution (40), in which the initial distribution is generated according to (17). The result (43) is an entropic uncertainty relation for a general SIC-POVM in the model of detection inefficiencies. The inefficiency-free lower bound (34) is multiplied by factor $\eta^{\alpha}$ and also added by the binary entropy $h_{\alpha}(\eta)$. Thus, an additional uncertainty is caused by the detector.

Let us consider entropic bounds for a single general SIC-POVM in terms of Rényi's entropy. For $\alpha>0 \neq 1$, the Rényi $\alpha$-entropy of probability distribution $\left\{p_{j}\right\}$ is defined as [40]

$$
R_{\alpha}(p):=\frac{1}{1-\alpha} \ln \left(\sum_{j} p_{j}^{\alpha}\right)
$$

When $\alpha \rightarrow 1$, this expression is reduced to the standard Shannon entropy. The entropy (44) is a non-increasing function of order $\alpha$ [40]. Taking $\alpha=2$, the expression (44) gives the collision entropy

$$
R_{2}(p)=-\ln \left(\sum_{j} p_{j}^{2}\right)=-\ln C(p)
$$

Note that the collision entropy is closely related to the index of coincidence and the linear entropy. In the limit $\alpha \rightarrow \infty$, we have the so-called min-entropy

$$
R_{\infty}(p)=-\ln \left(\max p_{j}\right)
$$

The min-entropy is of particular interest in cryptography [41]. It is also related to the extrema of the discrete Wigner function [42]. Rényi-entropies uncertainty relations are significant in studying the connection between complementarity and uncertainty principles [43]. Using the Rényi entropy, the writers of [44] formulated tradeoff relations for a trace-preserving quantum operation. An extension of such trade-off relations in terms of the so-called unified entropies was discussed in [45]. For a SIC-POVM $\mathcal{N}=\left\{\mathrm{N}_{j}\right\}$, the entropy $R_{\alpha}(\mathcal{N} \mid \boldsymbol{\rho})$ is obtained by substituting the probabilities (17) into (44). We now consider lower bounds on this entropy.

Proposition 3 Let general SIC-POVM $\mathcal{N}$ be characterized by the parameter $a$ in the sense of (7). For $\alpha \in[2 ; \infty)$ and arbitrary density matrix $\boldsymbol{\rho}$, the Rényi $\alpha$-entropy satisfies the state-dependent bound

$$
\begin{aligned}
& R_{\alpha}(\mathcal{N} \mid \boldsymbol{\rho}) \geq \\
& \frac{\alpha}{2(\alpha-1)} \ln \left(\frac{d\left(d^{2}-1\right)}{\left(a d^{3}-1\right) \operatorname{tr}\left(\boldsymbol{\rho}^{2}\right)+d(1-a d)}\right) .
\end{aligned}
$$

Proof. For $\alpha \geq 2$ and arbitrary probability distribution, we write the inequality

$$
\left(\sum_{j} p_{j}^{\alpha}\right)^{1 / \alpha} \leq\left(\sum_{j} p_{j}^{2}\right)^{1 / 2}=C(p)^{1 / 2} .
$$

This inequality follows from theorem 19 of the book [20]. The function $x \mapsto(1-\alpha)^{-1} \ln x$ decreases for $\alpha>1$. Combining this with (44) and (48) further gives

$$
R_{\alpha}(p) \geq \frac{\alpha}{2(1-\alpha)} \ln C(p) .
$$

The formulas (18) and (49) completes the proof.

The formula (47) provides a state-dependent lower bound on the Rényi $\alpha$-entropy of probability distribution generated by a general SIC-POVM. Due to (30), we can rewrite the bound (34) in the form

$$
R_{\alpha}(\mathcal{N} \mid \boldsymbol{\rho}) \geq \frac{\alpha}{2(1-\alpha)} \ln \left(\frac{1}{d^{2}}+\frac{2\left(a d^{3}-1\right)}{d^{3}\left(d^{2}-1\right)}\|r\|_{2}^{2}\right)
$$

For $\alpha=2$, the inequality (47) gives a bound on the collision entropy written as

$$
R_{2}(\mathcal{N} \mid \boldsymbol{\rho}) \geq \ln \left(\frac{d\left(d^{2}-1\right)}{\left(a d^{3}-1\right) \operatorname{tr}\left(\boldsymbol{\rho}^{2}\right)+d(1-a d)}\right) .
$$

As the Rényi $\alpha$-entropy does not increase with $\alpha$, the bound (51) is valid for all Rényi's entropies of order $\alpha \in$ $(0 ; 2]$, including the Shannon-entropy case $(37)$. For $\boldsymbol{\rho}=$ $|\psi\rangle\langle\psi|$, the entropic bound (47) is reduced to its purestate form

$$
R_{\alpha}(\mathcal{N} \mid \psi) \geq \frac{\alpha}{2(\alpha-1)} \ln \left(\frac{d(d+1)}{a d^{2}+1}\right),
$$

where $\alpha \in[2 ; \infty)$. Due to $\operatorname{tr}\left(\boldsymbol{\rho}^{2}\right)<1$ and increase of the logarithm, the lower bound (52) is weaker than (47). The right-hand side of $(47)$ is maximal for $\boldsymbol{\rho}_{*}=\mathbb{1} / d$. It is easy to see that $p_{j}\left(\mathcal{N} \mid \boldsymbol{\rho}_{*}\right)=d^{-2}$ and, therefore, $R_{\alpha}\left(\mathcal{N} \mid \boldsymbol{\rho}_{*}\right)=2 \ln d$ for all $\alpha>0$. With the completely mixed state, the right-hand side of (51) actually gives the bound $2 \ln d$ for $\alpha \in(0 ; 2]$. In this sense, the derived bound (47) is tight for such values of $\alpha$. For $\alpha>2$, the result (47) is always approximate. In fact, it gives the inequality $R_{\infty}\left(\mathcal{N} \mid \boldsymbol{\rho}_{*}\right) \geq \ln d$, which contains only a half of the exact value $R_{\infty}\left(\mathcal{N} \mid \boldsymbol{\rho}_{*}\right)=2 \ln d$. A way to improve relations with the min-entropy was discussed in [25]. We now extend such a treatment to general SIC-POVMs.

Proposition 4 Let general SIC-POVM $\mathcal{N}$ be characterized by the parameter $a$ in the sense of (7). For arbitrary density matrix $\boldsymbol{\rho}$, the min-entropy satisfies the state-dependent bound

$$
\begin{aligned}
& R_{\infty}(\mathcal{N} \mid \boldsymbol{\rho}) \geq \\
& 2 \ln d-\ln \left(1+\sqrt{a d^{3}-1} \sqrt{\operatorname{tr}\left(\boldsymbol{\rho}^{2}\right) d-1}\right)
\end{aligned}
$$


Proof. In appendix A of the paper [25], we proved the following statement. If the $n$ positive numbers $x_{j}$ obey the conditions $\sum_{j=1}^{n} x_{j}=1$ and $\sum_{j=1}^{n} x_{j}^{2}=b^{2}$, then

$$
\max _{1 \leq j \leq n} x_{j} \leq \frac{1}{n}\left(1+\sqrt{n-1} \sqrt{n b^{2}-1}\right) .
$$

Substituting (18) instead of $b^{2}$ and $d^{2}$ instead of $n$ into (54), we finally obtain

$$
\max _{1 \leq j \leq d^{2}} p_{j}(\mathcal{N} \mid \boldsymbol{\rho}) \leq \frac{1}{d^{2}}\left(1+\sqrt{a d^{3}-1} \sqrt{\operatorname{tr}\left(\boldsymbol{\rho}^{2}\right) d-1}\right) .
$$

Combining (46) with (55) directly leads to (53), since the function $x \mapsto-\ln x$ decreases.

The lower bound (53) is clearly stronger than the limiting case $\alpha \rightarrow \infty$ of the right-hand side of (47). With the completely mixed state, the result (53) gives the tight bound $2 \ln d$ due to $\operatorname{tr}\left(\boldsymbol{\rho}_{*}^{2}\right)=d^{-1}$. The right-hand side of (53) increases as the quantity $\operatorname{tr}\left(\boldsymbol{\rho}^{2}\right)$ decreases. In other words, the more a state is mixed, the more the bound (53). Replacing $\operatorname{tr}\left(\boldsymbol{\rho}^{2}\right)$ with 1 , the right-hand side of (53) gives the lower bound for the pure-state case. For $a=d^{-2}$, the upper bound (55) on the maximal probability leads to the analogous bound for a usual SIC-POVM. This particular case was already discussed in [25].

\section{UNCERTAINTY RELATIONS OF THE MAASSEN-UFFINK TYPE}

In this section, we will discuss entropic uncertainty relations for a pair of general SIC-POVMs. Since the celebrated Heisenberg's result was published [46], many approaches to incompatibilities in quantum measurements have been proposed. Entropic uncertainty relations were studied in many important cases [33, 34]. Results of such a kind are mainly based on the Maasen-Uffink approach [47]. This approach has been developed with use of various entropic functions. Entropic bounds in terms of generalized entropies entropic bounds were utilized in studying many topics such as the case of conjugate observables $[48,49]$, quantifying number-phase uncertainties [50, 51], incompatibilities of anti-commuting observables [52] and reformulations in quasi-Hermitian models [53]. In the context of simultaneous measurements of complementary observables, uncertainty relations in terms of both the Tsallis and Rényi entropies are examined in [54]. The method of Maassen and Uffink uses the Riesz theorem. It therefore leads to lower bound on the sum of two entropies, whose orders obey a certain condition [48, 50]. Entropic inequalities for quantum tomograms of qudit states are examined in [55]. Recently, new universal approach to entropic uncertainty relations has been proposed $[56,57]$. Apparently, this approach will play a significant role in future research. We will formulate uncertainty relations for two general SIC-POVMs in terms of the Rényi and Tsallis entropies as well as their symmetrized versions. We have the following statement.
Proposition 5 Let $\mathcal{M}=\left\{\mathrm{M}_{i}\right\}$ and $\mathcal{N}=\left\{\mathrm{N}_{j}\right\}$ be general SIC-POVMs. To any density matrix $\boldsymbol{\rho}$, we assign the quantity

$$
g(\mathcal{M}, \mathcal{N} \mid \boldsymbol{\rho}):=\max _{1 \leq i, j \leq d^{2}} \frac{\left|\operatorname{tr}\left(\mathrm{M}_{i} \mathrm{~N}_{j} \boldsymbol{\rho}\right)\right|}{p_{i}(\mathcal{M} \mid \boldsymbol{\rho})^{1 / 2} p_{j}(\mathcal{N} \mid \boldsymbol{\rho})^{1 / 2}} .
$$

Let positive orders $\alpha$ and $\beta$ obey $1 / \alpha+1 / \beta=2$, and let $\mu=\max \{\alpha, \beta\}$. Then the corresponding Tsallis entropies satisfy the inequality

$$
H_{\alpha}(\mathcal{M} \mid \boldsymbol{\rho})+H_{\beta}(\mathcal{N} \mid \boldsymbol{\rho}) \geq \ln _{\mu}\left(g(\mathcal{M}, \mathcal{N} \mid \boldsymbol{\rho})^{-2}\right) .
$$

Under the same preconditions, the corresponding Rényi entropies satisfy the inequality

$$
R_{\alpha}(\mathcal{M} \mid \boldsymbol{\rho})+R_{\beta}(\mathcal{N} \mid \boldsymbol{\rho}) \geq-2 \ln g(\mathcal{M}, \mathcal{N} \mid \boldsymbol{\rho}) .
$$

The presented formulations (57) and (58) immediately follows from the results of section 3 of [58]. The quantity (56) explicitly depends on the pre-measurement density matrix $\boldsymbol{\rho}$. It is of certain interest to obtain the stateindependent form of entropic bounds [59]. For the entropic relations (57) and (58), a way to obtain such forms was considered in $[53,58]$. Using the Cauchy-Schwarz inequality for the Hilbert-Schmidt inner product and the inequality (4), we finally obtain

$$
\begin{aligned}
& g(\mathcal{M}, \mathcal{N} \mid \boldsymbol{\rho}) \leq \bar{f}(\mathcal{M}, \mathcal{N}) . \\
& \bar{f}(\mathcal{M}, \mathcal{N}):=\max _{1 \leq i, j \leq d^{2}}\left\|\mathrm{M}_{i}^{1 / 2}\right\|_{\infty}\left\|\mathrm{N}_{j}^{1 / 2}\right\|_{\infty} .
\end{aligned}
$$

Here, we used three formulas

$$
\begin{aligned}
& p_{i}(\mathcal{M} \mid \boldsymbol{\rho})=\left\|\mathrm{M}_{i}^{1 / 2} \sqrt{\boldsymbol{\rho}}\right\|_{2}^{2}, \quad p_{j}(\mathcal{N} \mid \boldsymbol{\rho})=\left\|\mathrm{N}_{j}^{1 / 2} \sqrt{\boldsymbol{\rho}}\right\|_{2}^{2}, \\
& \operatorname{tr}\left(\mathrm{M}_{i} \mathrm{~N}_{j} \boldsymbol{\rho}\right)=\left\langle\mathrm{M}_{i} \sqrt{\boldsymbol{\rho}}, \mathrm{N}_{j} \sqrt{\boldsymbol{\rho}}\right\rangle_{\mathrm{hs}} .
\end{aligned}
$$

Due to (60), we obtain the state-independent entropic bounds

$$
\begin{gathered}
H_{\alpha}(\mathcal{M} \mid \boldsymbol{\rho})+H_{\beta}(\mathcal{N} \mid \boldsymbol{\rho}) \geq \ln _{\mu}\left(\bar{f}(\mathcal{M}, \mathcal{N})^{-2}\right), \\
R_{\alpha}(\mathcal{M} \mid \boldsymbol{\rho})+R_{\beta}(\mathcal{N} \mid \boldsymbol{\rho}) \geq-2 \ln \bar{f}(\mathcal{M}, \mathcal{N})
\end{gathered}
$$

in which the parameters $\alpha, \beta$, and $\mu$ are defined as in Proposition 5 . We can also reformulate these uncertainty relations in terms of the parameters $a_{\mathcal{M}}$ and $a_{\mathcal{N}}$ defined according to (7). Using the inequality (5), we write

$$
\left\|\mathrm{M}_{i}\right\|_{\infty}\left\|\mathrm{N}_{j}\right\|_{\infty} \leq\left\|\mathrm{M}_{i}\right\|_{2}\left\|\mathrm{~N}_{j}\right\|_{2}=\sqrt{a_{\mathcal{M}} a_{\mathcal{N}}} .
$$

This inequality is always saturated with the usual SICPOVMs, when $a_{\mathcal{M}} a_{\mathcal{N}}=d^{-4}$. By positivity, we also have

$$
\bar{f}(\mathcal{M}, \mathcal{N})^{2}=\max _{1 \leq i, j \leq d^{2}}\left\|\mathrm{M}_{i}\right\|_{\infty}\left\|\mathrm{N}_{j}\right\|_{\infty} .
$$

Combining (65) with (64) finally gives

$$
g(\mathcal{M}, \mathcal{N} \mid \boldsymbol{\rho})^{-2} \geq \bar{f}(\mathcal{M}, \mathcal{N})^{-2} \geq a_{\mathcal{M}}^{-1 / 2} a_{\mathcal{N}}^{-1 / 2}
$$


Since the function $x \mapsto \ln _{\mu}(x)$ is increasing, the inequality (66) leads to entropic uncertainty relations

$$
\begin{aligned}
& H_{\alpha}(\mathcal{M} \mid \boldsymbol{\rho})+H_{\beta}(\mathcal{N} \mid \boldsymbol{\rho}) \geq \ln _{\mu}\left(a_{\mathcal{M}}^{-1 / 2} a_{\mathcal{N}}^{-1 / 2}\right), \\
& R_{\alpha}(\mathcal{M} \mid \boldsymbol{\rho})+R_{\beta}(\mathcal{N} \mid \boldsymbol{\rho}) \geq-\frac{1}{2}\left(\ln a_{\mathcal{M}}+\ln a_{\mathcal{N}}\right)
\end{aligned}
$$

which follow from (62) and (63), respectively. The parameters $a_{\mathcal{M}}$ and $a_{\mathcal{N}}$ range according to (10). For two usual SIC-POVMs, the right-hand sides of (67) and (68) take values $\ln _{\mu}\left(d^{2}\right)$ and $2 \ln d$ due to $a_{\mathcal{M}}=a_{\mathcal{N}}=d^{-2}$. For other general SIC-POVMs, these bounds are strictly stronger. They may increase up to $\ln _{\mu}\left(d^{3}\right)$ and $3 \ln d$, when $a_{\mathcal{M}}$ and $a_{\mathcal{N}}$ both reach $d^{-3}$. However, the latter does not lead to legitimate SIC-POVMs. Since a SICPOVM has $d^{2}$ different outcomes, its Tsallis and Renyi $\alpha$ entropies are bounded from above by $\ln _{\alpha}\left(d^{2}\right)$ and $2 \ln d$, respectively. For two general SIC-POVMs, the formulas (67) and (68) show that the sum of the two corresponding entropies is not less than the maximal possible value for one of them. Thus, we have obtained non-trivial entropic bound of the Maassen-Uffink type.

Finally, we discuss reformulations in terms of the symmetrized entropies. For a pair of observables, uncertainty relations in terms of symmetrized entropies were given in both the Rényi [48] and Tsallis formulations [60]. In [25], lower bounds on the sum of symmetrized entropies have been derived for mutually unbiased bases. We assume that the entropic orders obey $1 / \alpha+1 / \beta=2$. Using $s \in[0 ; 1)$, we parameterize the orders as

$$
\max \{\alpha, \beta\}=\frac{1}{1-s}, \quad \min \{\alpha, \beta\}=\frac{1}{1+s} .
$$

The symmetrized Tsallis and Rényi entropies are respectively defined by

$$
\begin{aligned}
\widetilde{H}_{s}(\mathcal{N} \mid \boldsymbol{\rho}): & =\frac{1}{2}\left(H_{\alpha}(\mathcal{N} \mid \boldsymbol{\rho})+H_{\beta}(\mathcal{N} \mid \boldsymbol{\rho})\right) \\
\widetilde{R}_{s}(\mathcal{N} \mid \boldsymbol{\rho}): & =\frac{1}{2}\left(R_{\alpha}(\mathcal{N} \mid \boldsymbol{\rho})+R_{\beta}(\mathcal{N} \mid \boldsymbol{\rho})\right)
\end{aligned}
$$

Taking $\mu=(1-s)^{-1}$, for the sum $\widetilde{H}_{s}(\mathcal{M} \mid \boldsymbol{\rho})+\widetilde{H}_{s}(\mathcal{N} \mid \boldsymbol{\rho})$ we have the three lower bounds (57), (62), and (67). Sim- ilarly, the lower bounds (58), (63), and (68) are all valid for the sum $\widetilde{R}_{s}(\mathcal{M} \mid \boldsymbol{\rho})+\widetilde{R}_{s}(\mathcal{N} \mid \boldsymbol{\rho})$. Using symmetrized entropies, we can extend bounds of the Maassen-Uffink type to more than two measurements. An example with several mutually unbiased bases has been analyzed in [25]. In principle, this idea could be applied to general SIC-POVMs.

\section{CONCLUSIONS}

We have reported some properties of general symmetric informationally complete POVMs. SIC-POVMs are of interest in various topics such as quantum state tomography and quantum cryptography. Thus, the presented results may be useful within quantum technologies. For a general SIC-POVM and arbitrary measured state, the index of coincidence of generated probability distribution is exactly calculated. This result is a generalization of the previous calculation for a rank-one SIC-POVM. The obtained index of coincidence is expressed in terms of dimensionality, the trace of squared density matrix, and a parameter characterizing the given SIC-POVM. The trace of squared density matrix is one of measures quantifying a degree of state impurity. The calculation of the index of coincidence leads to entropic uncertainty relations for a single general SIC-POVM. We have expressed state-dependent formulations in terms of both the Rényi and Tsallis entropies. These formulations are an extension of entropic relations previously given in [25]. The min-entropy uncertainty relation is separately considered. For a pair of general SIC-POVMs, we discussed uncertainty relations of the Maassen-Uffink type. Reformulations in terms of the symmetrized entropies are briefly considered. A new important approach to obtaining entropic uncertainty bounds with the use of majorization technique has recently been proposed in the papers $[56,57]$. It may be interesting to study uncertainty relations for general SIC-POVMs on the base of majorization techniques.

The author is grateful to Karol Życzkowski for useful correspondence and valuable comments. The author thanks anonymous referee for constructive criticism.
[1] Nielsen M A and Chuang I L 2000 Quantum Computation and Quantum Information (Cambridge: Cambridge University Press)

[2] Prugovečki E 1977 Int. J. Theor. Phys. 16321

[3] Busch P 1991 Int. J. Theor. Phys. 301217

[4] Renes J M, Blume-Kohout R, Scott A J, and Caves C M 2004 J. Math. Phys. 452171

[5] Chiribella G, D'Ariano G M, Perinotti P, and Sacchi M F 2004 Phys. Rev. A $\mathbf{7 0} 062105$

[6] D'Ariano G M, Perinotti P, and Sacchi M F 2004 J. Opt. B: Quantum Semiclass. Opt. 6 S497
[7] Appleby D M, Fuchs C A, and Zhu H 2013 Group theoretic, Lie algebraic and Jordan algebraic formulations of the SIC existence problem arXiv:1312.0555 [quant-ph]

[8] Słomczyński W and Szymusiak A 2014 Highly symmetric POVMs and their informational power arXiv:1402.0375 [quant-ph]

[9] Durt T, Englert B-G, Bengtsson I, and Życzkowski K 2010 Int. J. Quantum Inf. 8535

[10] Appleby D M, Dang H B, and Fuchs C A 2007 Symmetric informationally-complete quantum states as analogues to orthonormal bases and minimum-uncertainty 
states arXiv:0707.2071 [quant-ph]

[11] Scott A J 2006 J. Phys. A: Math. Gen. 3913507

[12] Klappenecker A, Rötteler M, Shparlinski I, and Winterhof A 2005 J. Math. Phys. 46082104

[13] Appleby D M 2007 Optics and Spectroscopy 103416

[14] Kalev A 2013 A geometrical relation between symmetric operators and mutually unbiased operators arXiv:1305.6044 [quant-ph]

[15] Kalev A and Gour G 2013 Construction of all general symmetric informationally complete measurements arXiv:1305.6545 [quant-ph]

[16] Watrous J 2011 Theory of Quantum Information (Waterloo: University of Waterloo) http://www.cs.uwaterloo.ca/ watrous/CS766/

[17] Watrous J 2005 Quantum Inf. Comput. 558

[18] Rastegin A E 2011 Quantum Inf. Process. 10123

[19] Rastegin A E 2012 J. Stat. Phys. 1481040

[20] Hardy G H, Littlewood J E, and Polya G 1934 Inequalities (London: Cambridge University Press)

[21] Peres A 1993 Quantum Theory: Concepts and Methods (Dordrecht: Kluwer)

[22] Spekkens R W 2008 Phys. Rev. Lett. 101020401

[23] Ferrie C and Emerson J 2008 J. Phys. A: Math. Theor. 41352001

[24] Wu S, Yu S, and Mølmer K 2009 Phys. Rev. A 79022104

[25] Rastegin A E 2013 Eur. Phys. J. D 67269

[26] Bengtsson I and Życzkowski K 2006 Geometry of Quantum States: An Introduction to Quantum Entanglement (Cambridge: Cambridge University Press)

[27] Harremoës P and Topsøe F 2001 IEEE Trans. Inf. Theory 472944

[28] Klappenecker A and Rötteler M 2005 Mutually unbiased bases are complex projective 2-designs arXiv:quant$\mathrm{ph} / 0502031$

[29] Kimura G and Kossakowski A 2005 Open Sys. Inf. Dyn. 12207

[30] Kimura G 2003 Phys. Lett. A 314339

[31] Byrd M S and Khaneja N 2003 Phys. Rev. A 68062322

[32] Życzkowski K and Sommers H-J 2003 J. Phys. A: Math. Gen. 3610115

[33] Wehner S and Winter A 2010 New J. Phys. 12025009

[34] Białynicki-Birula I and Rudnicki Ł 2011 Entropic uncer- tainty relations in quantum physics Statistical Complexity (Berlin: Springer) pp 1-34

[35] Tsallis C 1988 J. Stat. Phys. 52479

[36] Furuichi S 2006 J. Math. Phys. 47023302

[37] Rastegin A E 2012 Kybernetika 48242

[38] Rastegin A E 2014 Quantum Inf. Comput. 140996

[39] Chaves R and Fritz T 2012 Phys. Rev. A 85032113

[40] Rényi A 1961 On measures of entropy and information Proc. of 4th Berkeley Symp on Mathematical Statistics and Probability (Berkeley, CA: University of California Press) pp 547-61

[41] Ng H Y N, Berta M, and Wehner S 2012 Phys. Rev. A 86042315

[42] Mandayam P, Wehner S, and Balachandran N $2010 \mathrm{~J}$. Math. Phys. 51082201

[43] Bosyk G M, Portesi M, Holik F, and Plastino A 2013 Phys. Scr. 87065002

[44] Roga W, Puchała Z, Rudnicki Ł, and Życzkowski K 2013 Phys. Rev. A 87032308

[45] Rastegin A E 2013 J. Phys. A: Math. Theor. 46285301

[46] Heisenberg W 1927 Z. Phys. 43172

[47] Maassen H and Uffink J B M 1988 Phys. Rev. Lett. 60 1103

[48] Białynicki-Birula I 2006 Phys. Rev. A 74052101

[49] Portesi M and Plastino A 1996 Physica A 225412

[50] Rastegin A E 2012 Quantum Inf. Comput. 120743

[51] Rastegin A E 2011 Phys. Scr. 84057001

[52] Wehner S and Winter A 2008 J. Math. Phys. 49062105

[53] Rastegin A E 2012 J. Phys. A: Math. Theor. 45444026

[54] Luis A 2013 Contradictory entropic joint uncertainty relations for complementary observables in two-level systems arXiv:1306.5211 [quant-ph]

[55] Man'ko M A and Man'ko V I 2013 J. Russ. Laser Res. $\mathbf{3 4 2} 203$

[56] Friedland S, Gheorghiu V, and Gour G 2013 Phys. Rev. Lett. 111230401

[57] Puchała Z, Rudnicki Ł, and Życzkowski K 2013 J. Phys. A: Math. Theor. 46272002

[58] Rastegin A E 2011 J. Phys. A: Math. Theor. 44095303

[59] Deutsch D 1983 Phys. Rev. Lett. 50631

[60] Rajagopal A K 1995 Phys. Lett. A 20532 